\title{
Successful radiofrequency ablation of accessory pathway associated with left atrial appendage aneurysm in a low birthweight premature patient
}

\author{
Yakup Ergül, Erkut Öztürk, Senem Özgür \\ Department of Pediatric Cardiology, Istanbul Mehmet Akif Ersoy Thoracic and Cardiovascular Surgery Center and Research \\ Hospital, Istanbul Sağlk Bilimleri University, Istanbul, Turkey. E-mail: erkut_ozturk@yahoo.com \\ Received: 25th August 2017, Revised: 26th September 2017, Accepted: 30th October 2017
}

SUMMARY: Ergül Y, Öztürk E, Özgür S. Successful radiofrequency ablation of accessory pathway associated with left atrial appendage aneurysm in a low birthweight premature patient. Turk J Pediatr 2019; 61: 142-146.

Tachyarrhythmias are common cardiac arrhythmias in newborns and infants. We present a premature case with a $2.4-\mathrm{kg}$ birth weight, with non-immune hydrops due to supraventricular tachycardia. Despite the combination of amiodarone, esmolol and flecainide, tachyarrhythmia could not be controlled and radiofrequency ablation was applied due to left ventricular dysfunction. After the procedure the rhythm returned to normal sinus and left ventricular functions improved rapidly. The follow-up of the case continues without any problems. In this presentation, successful ablation of the accessory pathway associated with structural heart disease have been covered in the youngest and smallest patient to date.

Key words: left atrial appendage aneurysm, newborn, radiofrequency ablation, supraventricular tachycardia.

Supraventricular tachycardia (SVT) is the most common type of tachyarrhythmia in pediatric cases. ${ }^{1}$ In newborns and infants, the majority of SVT are caused by the accessory pathway, and most do not repeat after one year of age. ${ }^{1}$ However, congestive heart failure findings may develop more easily during this period depending on the duration, mechanism, and speed of the tachycardia., ${ }^{1,2}$

In neonates and infants, the majority of SVTs were treated with single or multiple combinations of different antiarrhythmic drugs, taking spontaneous regression into account. ${ }^{2}$ It was reported that radiofrequency ablation (RFA) or cryoablation treatment had been performed successfully, yet these procedures had a higher risk than other age groups in a limited number of medical treatment-resistant patients. 3,4

In this article, we present a newborn case weighing $2.4 \mathrm{~kg}$ having non-immune hydrops fetalis due to fetal tachyarrhythmia associated with left atrial appendage aneurysm. This case is the youngest patient with the lowest weight to date, with successful RFA of the accessory pathway related to the structural heart pathology.

\section{Case Report}

A 30-day-old male patient was referred to our clinic due to persistent tachycardia despite multiple antiarrhythmic treatment. It was discovered from the history that the mother was admitted to hospital and a cesarean section was performed at the 34th gestational week because of non-immune hydrops fetalis. In order to treat incessant tachyarrhythmia and to monitor signs of heart failure, the baby was transferred to the neonatal intensive care unit NICU. Amiodarone infusion, esmolol infusion, and flecainide treatment were started because of tachyarrhythmia attacks in the NICU. In the early stages, tachyarrhythmia was partially controlled by adenosine and antiarrhythmic therapy. However, it could not be controlled. The patient was referred to our clinic for follow-up and treatment of tachyarrhythmia.

The newborn's reflexes were depressed, and 


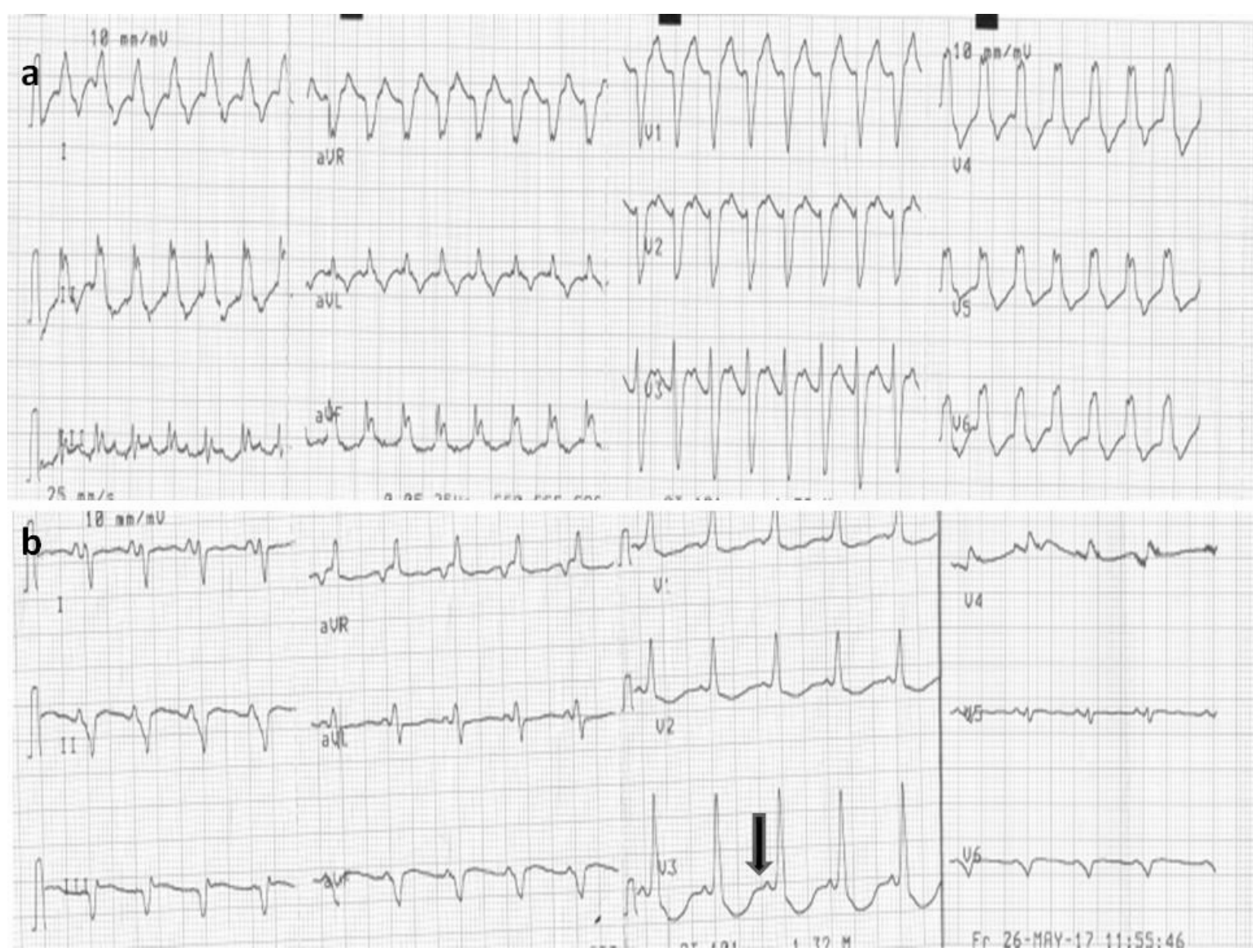

Fig. 1.

1a. The 12 derivation ECG recording of the wide QRS tachycardia of the patient. A broad QRS tachycardia with left bundle branch block was noticed via an electrocardiography (ECG) with P waves undetectable at $200 \mathrm{~min}$.

1b. Wolff-Parkinson-White (WPW) recording of the patient after cessation of tachycardia. The arrow shows an example of WPW characterized by the short PR interval, wide QRS and delta wave

suction was weak at the time of admission. On physical examination, tachypnea, dyspnea, tachycardia, 2/6 systolic murmur at apex, and $3 \mathrm{~cm}$ hepatomegaly were detected. A broad QRS tachycardia with left bundle branch block was noticed via an electrocardiography (ECG) with $P$ waves undetectable at 200 min. (Fig. 1a). Echocardiography revealed left atrial appendage aneurysm, left ventricular dilatation (left ventricular end-diastolic diameter: 21 $\mathrm{mm}, \mathrm{z}$ value: 2.25 ), mild-moderate mitral regurgitation, and patent foramen ovale. Left ventricular systolic functions were depressed. Initially, ejection fraction and shortening fraction were measured as $44 \%$ and $20 \%$, respectively. When adenosine was given, the tachycardia stopped for a short while and then restarted. When the tachycardia stopped, intermittent Wolff-Parkinson-White syndrome became visible (Fig. 1b). The treatment continued with amiodarone (10 mg/kg/day), esmolol (150 microgram/kg/day), and flecainide (100 mg/ $\mathrm{m}^{2} /$ day). Despite the aggressive antiarrhythmic treatment, hemodynamic instability continued and lactate levels in blood gas increased at the 16th hour of follow-up. At that point, it was decided that the patient should be taken urgently for an RFA process. Written informed consent was obtained from the patient's family.

The patient was transferred to the electrophysiologic study (EPS) laboratory. A 5 -F sheath was inserted into both the right jugular and right femoral veins. A four-electrode transesophageal catheter was also used for diagnostic purposes. A right jugular EPS catheter was advanced to the right ventricular apex. An ablation catheter was placed from the right femoral region. During broad QRS tachycardia, the earliest ventriculoatrial (VA) 

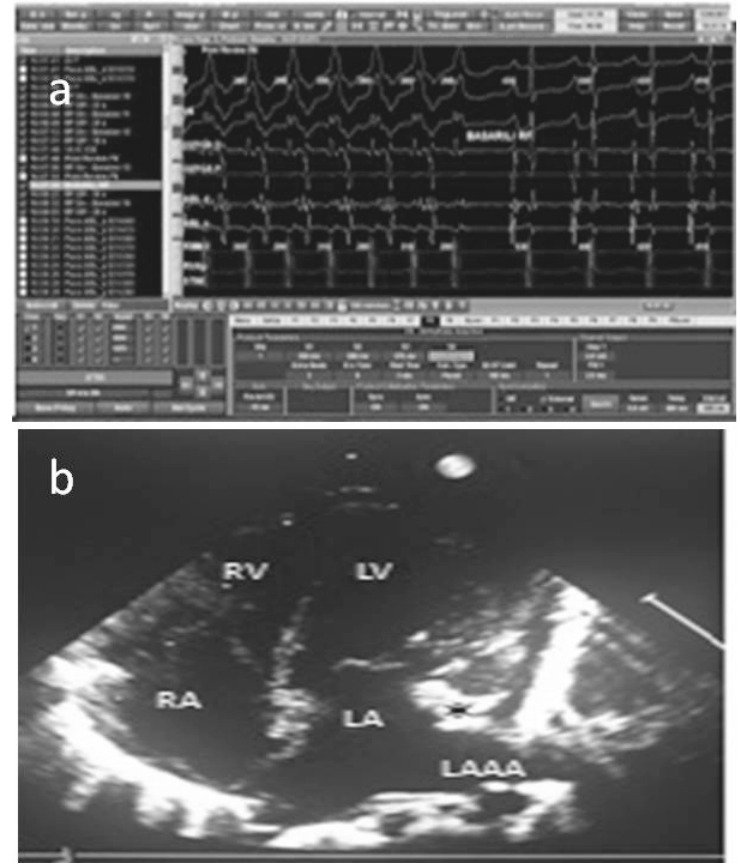

Fig. 2.

2a. The cessation of tachycardia after RF ablation.

2b. Hyperechogenic focus at 4-chamber view on echocardiographic examination.

* hyperechogenic focus, RA: right atrium, LA: left atrium, RV: right ventricle, LV: left ventricle, LAAA: left atrial appendage aneurysm

conduction time was observed at the distal tip of the esophagus. During right atrium mapping, any measurement which was earlier than the distal esophagus could not be obtained. It was seen that the earliest of the VA conduction times was in the left lateral region with 37 msec.

The 5-F RF ablation catheter terminated tachycardia with VA block at 2.2 seconds of the RF lesion, which started with 13-20 watts of power and $40-50{ }^{\circ} \mathrm{C}$ heat at the earliest receiving site (Figure 2a). After a total of three RF lesions, the rhythm became sinus and pre-excitation was not detected on ECG. During sinus rhythm, there were high amplitude $\mathrm{P}$ waves representing left atrial dilatation. After 30 minutes, SVT could not be created with programmed atrial stimuli. Despite the orciprenaline flush, VA dissociated at $380 \mathrm{msec}$ by ventricular pacing. The left ventricular function was measured as normal in the echocardiography performed the following day. Aspirin (PO) was initiated due to entrance of the left atrium and ventricle. The patient was discharged without any complications on the third day after admission. One week later, at first check-in, the left ventricular function of the patient was still normal, and on the left atrium appendage aneurysm, radiofrequency received regions were detected as hyperechogenic focuses (Fig. 2b). At the two-month follow-up, the patient still had no problems.

\section{Discussion}

The most common type of tachyarrhythmia in children under one year of age is supraventricular tachycardia; $80 \%$ of SVTs are atrioventricular reentrant tachycardia (AVRT). ${ }^{1}$ SVT is usually seen in structurally normal hearts, however, about $13 \%$ of SVTs are detected in patients with congenital heart disease. The top three most common diseases associated with SVT are ventricular septal defect, atrial septal defect, and Ebstein's anomaly, respectively. ${ }^{2}$

Left atrial appendage aneurysm (LAAA) is a rare structural anomaly associated with congenital dysplasia of pectinate muscles. ${ }^{5}$ Very rarely, mitral valve acquired inflammation and degenerative deformation may be the cause. Histologic studies have shown that aneurysms include three heart layers but myocardium is very thin and fibrotic. ${ }^{6}$ There is an increased association with pericardial defects.

LAAAs have been reported in all age groups from the fetal to elderly period ${ }^{6,7}$, but usually present in the $20 \mathrm{~s}$ to $40 \mathrm{~s} .{ }^{7}$ The reason for this is the nature of the aneurysm itself, which is generally small at the beginning and grows gradually. Consequently, it tends to remain asymptomatic in the early stages of life if no complications occur. Patients usually present with atrial tachyarrhythmias as a result of ectopic foci of atrial rhythm generation, systemic thromboembolism. More rarely, aneurysm can lead to cardiac failure, mitral regurgitation, and ischemic symptoms via impairing synchronization or compression of vital structures such as coronaries. ${ }^{8,9}$

In patients with atrial aneurysm, SVT is most commonly associated with ectopic atrial focal and intra-atrial re-entry. ${ }^{10}$ In fact, many authors claim that the frequency of LAAA increases in adults with atrial fibrillation compared to the normal population. Conversely, in our young patient, the arrhythmia mechanism was preexcitation. It is thought that these accessory 
pathways are caused by direct contact between the aneurysm and the left ventricle. The aneurysm can by-pass the annulus and form a functional accessory pathway.

If the first finding of LAAA is arrhythmia, surgical excision is widely accepted to treat both arrhythmia and to avoid thromboembolism and compression that might arise during future follow-up. Since our patient was low-weight, premature, and hemodynamically unstable, a surgical option could not have been considered as a first-step management.

In fact, in the fetal period, SVTs are very rare but can lead to heart failure resulting in hydrops fetalis. ${ }^{11}$ In the fetal/newborn period, the course of SVT is usually benign and can be controlled with antiarrhythmic drugs. However, about 7\% of cases show resistance to multiple medical combinations. ${ }^{12}$ RFA should be considered in case of arrhythmias which are long-standing and unresponsive to medical therapy. In our patient, sinus conversion was not achieved despite the beta blocker, group IC, and group 3 antiarrhythmic therapies.

RFA in young children has several difficulties. ${ }^{13}$ The small size of the heart and the anatomic variation may adversely affect ablation success in infants weighing less than $15 \mathrm{~kg} .{ }^{14}$ The left atrial aneurysm could adversely affect both ablation response and access to the target area. Another problem is that newborns and infants have limited cardiac reserves, and compensation mechanisms are directly related to heart rate. SVTs are likely to be longterm because infants cannot express their symptoms. ${ }^{15}$ Hence, incessant tachycardia can easily disrupt hemodynamics, leading hemodynamic instability of the patient to be an additional risk factor in terms of procedural success. ${ }^{13-15}$ Our patient was also treated with RFA in a very unstable condition.

In premature infants with low birth weight, the rate of complications related to the procedure is high. This is due to relatively stiff catheters and small cardiac volume. Therefore, critical structures such as AV node and coronary artery can easily be damaged during catheter maneuvers. Additionally, the newborn's cardiac tissue is more vulnerable to damage during therapeutic energies. ${ }^{15}$ Uncontrolled spread of radiofrequency energy in the immature heart and enlargement of the lesion may increase morbidity. Choosing general anesthesia, transesophageal probe insertion instead of HRA catheter, formation of the lesion on the atrial side RFA with limited heat, energy, and time, and using catheters as small as possible may reduce complication rates.

In conclusion, neonatal and infant tachyarrhythmias greatly differ in terms of clinical and arrhythmia subtype. Ablation is an effective alternative therapy for patients with resistance to medical therapy and/or left ventricular dysfunction. Although RFA is widely accepted in infants and young children, experience is very limited in very low birth weight premature patients even in wellequipped reference centers ${ }^{16}$. In the literature, we have not found successful RFA of SVT related to structural heart abnormality within this weight range. In this article, successful RFA of the accessory pathway related to the left atrial appendage diverticulum in the youngest and smallest patient was presented for the first time.

\section{REFERENCES}

1. Guerrier K, Shamszad P, Czosek RJ, Spar DS, Knilans TK, Anderson JB. Variation in antiarrhytmic management of infants hospitalized with supraventricular tachycardia: A multi-institutional analysis. Pediatr Cardiol 2016; 37:946-952.

2. Chu PY, Hill KD, Clark RH, Smith PB, Hornik CP. Treatment of supraventricular tachycardia in infants: Analysis of a large multicenter database. Early Hum Dev 2015; 91:345-350.

3. Akdeniz C, Ergul Y, Kıplapinar N, Tuzcu V. Catheter ablation of drug resistant supraventricular tachycardia in neonates and infants. Cardiol J 2013; 20:241-246.

4. Svintsova LI, Popov SV, Kovalev IA. Radiofrequency ablation of drug-refractory arrhytmias in small children younger than 1 year of age: Single center experience. Pediatr Cardiol 2013; 34:1321-1329.

5. Wilson D, Kalra N, Brody EA, Dyk H, Sorrell VL. Left atrial appendage aneurysm-a rare anomaly with an atypical presentation. Congenit Heart Dis 2009; 4:489-493.

6. Nagai T, Higaki J, Okayama H. Atrial tachycardia in congenital left atrial appendage aneurysm: Threedimensional computed tomography imaging with electro-anatomical mapping. Eur Heart J 2010; 31 : 1590 .

7. Cho MJ, Park J, Lee HD, Choo KS, Sung SI. Congenital left atrial appendage aneurysm diagnosed by fetal echocardiography. J Clin Ultrasound 2010; 38: 94-96. 
8. Krueger SK, Ferlic RM, Mooring PK. Left atrial appendage aneurysm: correlation of noninvasive with clinical and surgical findings: Report of a case. Circulation 1975; 52: 732-738.

9. Haydın S, Guzeltaş A, Ozturk E, Odemıs E, Bakır I. Huge aneurysm of the left atrial appendage in childhood. Turk Gogus Kalp Damar Cerrahisi Dergisi 2014; 22: 171-73.

10. Shah K, Walsh K. Giant right atrial diverticulum: An unusual cause of Wolff-Parkinson-White syndrome. Br Heart J 1992; 68: 58-59.

11. Van Engelen AD, Wiejtens O, Brener JI, et al. Management, outcome and follow-up of fetal tachycardia. J Am Coll Cardiol 1994; 24: 1371-1375.

12. Manole MD, Saladino R. Emergency department management of the pediatric patient with supraventricular tachycardia. Pediatr Emerg Care 2007; 23: 176-185.
13. Weindling SN, Saul JP, Walsh EP. Efficacy and risks of medical therapy for supraventricular tachycardia in neonates and infants. Am Heart J 1996; 131: 66-72.

14. Kugler JD, Danford DA, Houston K, Felix G. Radiofrequency catheter ablation for paroxysmal supraventricular tachycardia in children and adolescents without structural heart disease. Am J Cardiol 1997; 80: 1438-1443.

15. Saul JP. Special Considerations for Ablation in Pediatric Patients. In: Miller JM (ed). Catheter Ablation of Cardiac Arrhythmias (3rd ed). Philadelphia: Saunders, 2015: 718-748.

16. Femenia F, Sarquella-Brugada G, Brugada J. Singlecatheter radiofrequency ablation of a permanent junctional reciprocating tachycardia in a premature neonate. Cardiol Young 2012; 22: 606-609. 